\title{
Improving Application of Bayesian Neural Networks to Discriminate Neutrino Events from Backgrounds in Reactor Neutrino Experiments
}

\author{
Ye Xur, WeiWei Xut ${ }^{a}$, YiXiong Meng ${ }^{a}$, Bin Wu $u^{a}$ \\ ${ }^{a}$ Department of Physics, Nankai University, Tianjin 300071, China
}

\begin{abstract}
The application of Bayesian Neural Networks(BNN) to discriminate neutrino events from backgrounds in reactor neutrino experiments has been described in Ref.[1]. In the paper, BNN are also used to identify neutrino events in reactor neutrino experiments, but the numbers of photoelectrons received by PMTs are used as inputs to BNN in the paper, not the reconstructed energy and position of events. The samples of neutrino events and three major backgrounds from the Monte-Carlo simulation of a toy detector are generated in the signal region. Compared to the BNN method in Ref.[1], more ${ }^{8} \mathrm{He} /{ }^{9} \mathrm{Li}$ background and uncorrelated background in the signal region can be rejected by the BNN method in the paper, but more fast neutron background events in the signal region are unidentified using the BNN method in the paper. The uncorrelated background to signal ratio and the ${ }^{8} \mathrm{He} /{ }^{9} \mathrm{Li}$ background to signal ratio are significantly improved using the BNN method in the paper in comparison with the BNN method in Ref.[1]. But the fast neutron background to signal ratio in the signal region is a bit larger than the one in Ref.[1].
\end{abstract}

Keywords: Bayesian neural networks, neutrino oscillation, identification

PACS numbers: 07.05.Mh, 29.85.Fj, 14.60.Pq

\section{Introduction}

The main goals of reactor neutrino experiments are to detect $\overline{\nu_{e}} \rightarrow \overline{\nu_{x}}$ oscillation and precisely measure the mixing angle of neutrino oscillation $\theta_{13}$. The experiment is designed to detect reactor $\overline{\nu_{e}}$ 's via the inverse $\beta$-decay reaction

$$
\overline{\nu_{e}}+p \rightarrow e^{+}+n .
$$

The signature is a delayed coincidence between $e^{+}$and the neutron captured signals. In the paper, only three important sources of backgrounds are taken into account and they are the uncorrelated background from natural radioactivity and

\footnotetext{
${ }^{*}$ Corresponding author, e-mail address: xuye76@nankai.edu.cn
} 
the correlated backgrounds from fast neutrons and ${ }^{8} \mathrm{He} /{ }^{9} \mathrm{Li}$. The backgrounds like the neutrino events consist of two signals, a fast signal and a delay signal. It is vital to separate neutrino events from backgrounds accurately in the reactor neutrino experiments. Bayesian neural networks (BNN)[2] are algorithms of the neural networks trained by Bayesian statistics. They are not only non-linear functions as neural networks, but also controls model complexity. So their flexibility makes them possible to discover more general relationships in data than traditional statistical methods and their preferring simple models make them possible to solve the over-fitting problem better than the general neural networks[3]. BNN have been used to particle identification and event reconstruction in the experiments of the high energy physics, such as Ref. $[1,4,5,6]$. The application of BNN to discriminate neutrino events from backgrounds in reactor neutrino experiments has been described in Ref.[1]. In the paper, BNN are also used to identify neutrino events in the signal region[1] in reactor neutrino experiments, but the numbers of photoelectron received by PMTs are used as inputs to BNN, not the reconstructed energy and position of events.

\section{The Classification with $\mathrm{BNN}[1,2,6]$}

The idea of BNN is to regard the process of training a neural network as a Bayesian inference. Bayes' theorem is used to assign a posterior density to each point, $\bar{\theta}$, in the parameter space of the neural networks. Each point $\bar{\theta}$ denotes a neural network. In the methods of BNN, one performs a weighted average over all points in the parameter space of the neural network, that is, all neural networks. The methods are described in detail in Ref.[1, 2, 6]. The posterior density assigned to the point $\bar{\theta}$, that is, to a neural network, is given by Bayes' theorem

$$
p(\bar{\theta} \mid x, t) \propto p(t \mid x, \bar{\theta}) p(\bar{\theta})
$$

Where $x$ is a set of input data which corresponds to a set of target $t$. The likelihood $p(t \mid x, \bar{\theta})$ can be obtained by using a training sample. And a Gaussian prior is specified for each weight using the Bayesian neural networks package of Radford $\mathrm{Neal}^{1}$. Given an event with data $x^{\prime}$, an estimate of the probability that it belongs to the signal is given by the weighted average

$$
\bar{y}\left(x^{\prime} \mid x, t\right)=\int y\left(x^{\prime}, \bar{\theta}\right) p(\bar{\theta} \mid x, t) d \bar{\theta}
$$

Currently, the only way to perform the high dimensional integral in Eq. (2) is to sample the density $p(\bar{\theta} \mid x, t)$ with Markov Chain Marlo Carlo (MCMC) methods $[2,7,8,9]$. In MCMC methods, one steps through the $\bar{\theta}$ parameter space in such a way that points are visited with a probability proportional to the posterior density, $p(\bar{\theta} \mid x, t)$. Points where $p(\bar{\theta} \mid x, t)$ is large will be visited more often than points where $p(\bar{\theta} \mid x, t)$ is small.

\footnotetext{
${ }^{1}$ R. M. Neal, Software for Flexible Bayesian Modeling and Markov Chain Sampling, http://www.cs.utoronto.ca/ radford/fbm.software.html
} 
Eq. (2) approximates the integral using the average

$$
\bar{y}\left(x^{\prime} \mid x, t\right) \approx \frac{1}{L} \sum_{i=1}^{L} y\left(x^{\prime}, \bar{\theta}_{i}\right)
$$

where $L$ is the number of points $\bar{\theta}$ sampled from $p(\bar{\theta} \mid x, t)$. Each point $\bar{\theta}$ corresponds to a different neural network with the same structure. So the average is an average over neural networks, and the probability of the data $x^{\prime}$ belongs to the signal. The average is closer to the real value of $\bar{y}\left(x^{\prime} \mid x, t\right)$, when $L$ is sufficiently large.

\section{Toy Detector and Monte-Carlo Simulation[5]}

In the paper, a toy detector is used to simulate central detectors in the reactor neutrino experiments, such as Daya Bay experiment[10] and Double Chooz experiment[11], with CERN GEANT4 package[12]. The toy detector is the same as Ref.[5]. A total of 366 PMTs are arranged in 8 rings of 30 PMTs on the lateral surface of the oil region, and in 5 rings of 24, 18, 12, 6, 3 PMTs on the top and bottom caps.

The responses of neutrino events and backgrounds deposited in the toy detector are simulated with GEANT4. Although the physical properties of the scintillator and the oil (their optical attenuation length, refractive index and so on) are wavelength dependent, only averages[13] (such as the optical attenuation length of Gd-LS with a uniform value is 8 meter and the one of LS is 20 meter) are used in the detector simulation.

According to the anti-neutrino interaction in detectors of the reactor neutrino experiments[14], a neutrino event is uniformly generated throughout Gd-LS region (see Fig. 1). A uncorrelated background event is generated in such a way that a $\gamma$ event generated on the base of the energy distribute of the natural radioactivity in the proposal of the Day Bay experiment[10] is regarded as the fast signal, a neutron event of the single signal is regarded as the delay signal, its delay time is uniformly generated from $2 \mu \mathrm{s}$ to $100 \mu \mathrm{s}$ and the positions of the fast signal and the delay signal are uniformly generated throughout Gd-LS region. A fast neutron event is uniformly generated throughout Gd-LS region and its energy are uniformly generated from $0 \mathrm{MeV}$ to $50 \mathrm{MeV}$, therein an event of two signals are regarded as a fast neutron background event. Since the behaviors of ${ }^{8} \mathrm{He} /{ }^{9} \mathrm{Li}$ decay events in detectors couldn't be simulated by the Geant4 package, a ${ }^{8} \mathrm{He} /{ }^{9} \mathrm{Li}$ event is generated in such a way that the neutron signal from a fast neutron event is regarded as its delay signal, an electron event generated at the same position as the fast neutron event on the base of the energy distribute of ${ }^{8} \mathrm{He} /{ }^{9} \mathrm{Li}$ events in the proposal of the Day Bay experiment[10] is regarded as its fast signal in the paper.

Energies and positions of neutrino events and backgrounds are reconstructed by the method in Ref.[5]. The signal region is determined by using the reconstructed energies and positions, as well as the neutron delay time(described in Ref.[1]). 


\section{Neutrino Discrimination with BNN}

Choosing inputs to BNN is vital to identify neutrino events. The reconstructed energies, the distance between reconstructed the positions of neutron and positron and the neutron delay time were used as inputs to the BNN method in Ref.[1], but the energies and the distance are both the reconstructed physics variables, and they make BNN discriminations worse because of their reconstruction uncertainties. So we try to use raw data as inputs to BNN. Obviously, the numbers of photoelectrons received by 366 PMTs are rawer than the reconstructed variables. An event consists of two signals (a fast signal and a delay signal), so if the numbers of photoelectron received by PMTs will be directly used as inputs to BNN, BNN will have 732 inputs at least. It will take too much time to run a BNN program in a general computer because of such many inputs. The method of reducing inputs to BNN in the paper is that the photoelectrons received by several neighboring PMTs are added up. That is several neighboring PMTs incorporate a PMT patch. In the paper, a PMT patch is a 3 (azimuth direction) $\times 4$ (z direction) PMTs array on the detector lateral surface or a $120^{\circ}$ sector(including 21 PMTs) on the detector top and bottom caps. The delay time between two signals is very important to discriminate neutrino events from the uncorrelated background, so the number of photoelectrons received by a patch is multiply by the delay time, and the result is used as the inputs to all neural networks, which have the same structure. Then all the networks have a input layer of 52 inputs, the single hidden layer of fifteen nodes and a output layer of a single output which is just the probability that an event belongs to the neutrino event. Discriminating neutrino events from backgrounds is actually a binary response problem, that is the target is ' 1 ' or ' 0 '. Neutrino events are labeled by $\mathrm{t}=1$, and background events are labeled by $\mathrm{t}=0$. So the output of $\mathrm{BNN}$ has to be a number between 0 and 1 . If the output is less than 0.5 , the event is regarded as a background event, and If the output is larger than 0.5 , the event is regarded as a neutrino event.

A Markov chain of neural networks is generated using the Bayesian neural networks package of Radford Neal, with a training sample consisting of neutrino events and background events. One thousand iterations, of twenty MCMC steps each, are used. The neural network parameters are stored after each iteration, since the correlation between adjacent steps is very high. That is, the points in neural network parameter space are saved to lessen the correlation after twenty steps here. It is also necessary to discard the initial part of the Markov chain because the correlation between the initial point of the chain and the point of the part is very high. The initial three hundred iterations are discarded here. It takes about 120 hours to run 1000 iterations on a computer with two $3.4 \mathrm{GHz}$ Intel Pentium D processors (only one of which are used).

Neutrino identification efficiencies are defined by the ratios between the number of the events in neutrino test sample regarded as neutrinos and the number of neutrino test sample. Background identification efficiencies are defined by the ratios between the numbers of the events in background test samples regarded as background events and the numbers of background test samples. The identification efficiencies are measured with the test sample which is different from the training sample. Other 3000 events each of the neutrino and the three backgrounds are used to test the identification capability of the trained BNN. In the paper, BNN are trained by the different training samples, which consist of neutrinos and three backgrounds at different rates, since the different identification efficiencies 
are obtained using those BNN.

\section{Results and Discussion}

As Tab. 1 shows, most neutrino events, uncorrelated background events and ${ }^{8} \mathrm{He} /{ }^{9} \mathrm{Li}$ background events in the signal region can be identified using the BNN method in the paper, but only a small part of fast neutron background events can be identified using the BNN method in the paper. Since most fast neutron events can't be discriminate from neutrino events using the BNN method in the paper, neutrino discriminations are concerned with neutrino rates in training samples, as well as ratios of neutrino events and fast neutron events in training samples. The neutrino discrimination in the signal region increases from $90.5 \%$ to $93.7 \%$ with the increase of the neutrino rate from $50.0 \%$ to $57.1 \%$ in the training sample using the BNN method in the paper. And the neutrino discrimination also increases from $90.5 \%$ to $94.1 \%$ with the increase of the ratio of neutrino events and fast neutron events from 2:1 to $3: 1$ in the training sample. The different background to signal ratios in the signal region are obtained using the BNN trained by the training samples consisting of neutrino events and background events at different rates in the reactor neutrino experiments.

Neutrino events are discriminated from fast neutrons and ${ }^{8} \mathrm{He} /{ }^{9} \mathrm{Li}$ events via their fast signals identification, that is positron signals from neutrino events are separated from recoil proton signals from fast neutrons and electron signals from ${ }^{8} \mathrm{He} /{ }^{9} \mathrm{Li}$ events. $\gamma$ signals induced by positrons and recoil protons are closer to point sources, but $\gamma$ signals induced by electrons are closer to line sources. There is an effect on the distribution of photoelectrons over all the PMTs in the detector due to the difference between a point source and a line source. The effect can be extracted from the inputs by the BNN method in the paper. So neutrino events can be better discriminated from ${ }^{8} \mathrm{He} /{ }^{9} \mathrm{Li}$ events using the BNN method in the paper, but distinguishing between neutrinos events and fast neutrons becomes worse using the BNN method in the paper.

The events in the signal region can be identified using BNN one by one, once those BNN are trained by training samples. If the BNN method in the paper is used to the reactor neutrino experiments, the background to signal ratios will be changed. We only roughly estimate the changes here. We assume that the uncorrelated background fraction in the signal region is $A / N$, the fast neutrons background fraction in the signal region is $F / N$, and the ${ }^{8} \mathrm{He} /{ }^{9} \mathrm{Li}$ background fraction in the signal region is $L / N$ in the reactor neutrino experiments. Those background fractions are very low (for example, they are $<0.2 \%, 0.1 \%, 0.3 \%$ in one of the near detector claimed by the proposal of the Daya Bay experiment[10], respectively). If neutrino events are discriminated from background events using the BNN method in Ref.[1], the background to signal ratios can reach $0.2^{*}(\mathrm{~A} / \mathrm{N})$, $0.68^{*}(\mathrm{~F} / \mathrm{N})$ and $0.66^{*}(\mathrm{~L} / \mathrm{N})$, respectively. If the efficiencies of the first column in Tab. 1 are use to the estimation, we get the result of the identification using the BNN method in the paper:

Uncorrelated background/Signal $=(\mathrm{A} / \mathrm{N}) *(1-0.983) / 0.941=0.018^{*}(\mathrm{~A} / \mathrm{N})$

Fast neutrons Background/Signal $=(\mathrm{F} / \mathrm{N}) *(1-0.293) / 0.941=0.751 *(\mathrm{~F} / \mathrm{N})$ ${ }^{8} \mathrm{He} /{ }^{9} \mathrm{Li}$ Background $/$ Signal $=(\mathrm{L} / \mathrm{N}) *(1-0.913) / 0.941=0.092 *(\mathrm{~L} / \mathrm{N})$

As the above equations show, the uncorrelated background to signal ratio and ${ }^{8} \mathrm{He} /{ }^{9} \mathrm{Li}$ background to signal ratio in the signal region are significantly improved 
using the BNN method in the paper in comparison with the BNN method in Ref.[1]. And the fast neutron background to signal ratio is a bit larger than the one in Ref.[1]. But the fast neutron fraction in the signal region is lower than the ones of the uncorrelated background and ${ }^{8} \mathrm{He} /{ }^{9} \mathrm{Li}$ background, so the total background to signal ratio using the BNN method in the paper is much lower than the one in Ref.[1]. In a word, the BNN method in the paper can be applied to discriminate neutrino events from background events better than the BNN method in Ref.[1] and the method based on the cuts in reactor neutrino experiments.

\section{Acknowledgements}

This work is supported in part by the National Natural Science Foundation of China (NSFC) under the contract No. 10605014, the national undergraduate innovative plan of China under the contract No.081005517 and the physical base of Nankai University under the contract No. J0730315.

\section{References}

[1] Y. Xu, Y. X. Meng, and W. W. Xu, Applying bayesian neural networks to separate neutrino events from backgrounds in reactor neutrino experiments, Journal of Instrumentation, 3, P08005 (2008), arXiv: 0808.0240

[2] R. M. Neal, Bayesian Learning of Neural Networks. New York: SpringerVerlag, 1996

[3] R. Beale and T. Jackson, Neural Computing: An Introduction, New York: Adam Hilger, 1991

[4] Y. Xu, J. Hou and K. E. Zhu, Applying Bayesian neural networks to identify pion, kaon and proton in BESII, Chinese Physics C32, 201-204 (2008)

[5] Y. Xu, W. W. Xu, Y. X. Meng, and W. Xu, Applying Bayesian neural networks to event reconstruction in reactor neutrino experiments, Nuclear Instruments and Methods in Physics Rearch A592, 451-455 (2008), arXiv: 0712.4042

[6] P. C. Bhat and H. B. Prosper Beyesian Neural Networks. In: L. Lyons and M. K. Unel ed. Proceedings of Statistical Problems in Particle Physics, Astrophysics and Cosmology, Oxford, UK 12-15, September 2005. London: Imperial college Press. 2006. 151-154

[7] S. Duane, A. D. Kennedy, B. J. Pendleton and D. Roweth, Hybrid Monte Carlo, Physics Letters, B195, 216-222 (1987)

[8] M. Creutz and A. Gocksch, Higher-order hybrid Monte Carlo, Physical Review Letters, 1989 63, 9-12

[9] P. B. Mackenzie, An improved hybrid Monte Carlo method, Physics Letters, 1989 B226, 369-371

[10] Daya Bay Collaboration, Daya Bay Proposal: A Precision Measurement of the Neutrino Mixing Angle $\theta_{13}$ Using Reactor Antineutrino At Daya Bay, arXiv: hep-ex/0701029 
[11] M. Goodman and T. Lasserre, Double Chooz: A Search for the Neutrino Mixing Angle $\theta_{13}$, arXiv: hep-ex/0606025

[12] Geant4 Reference Manual, vers. 9.0 (2007)

[13] The CHOOZ Experiment Proposal (1993), available at the WWW site http://duphy4.physics.drexel.edu/chooz_pub/

[14] Y. X. Sun, J. Cao, and K. J. Luk, et al., Baseline Optimization of Reactor Neutrino experiments, Chinese Physics C29, 543-548 (2005) 
Tab. 1: The different identification efficiencies are obtained with the BNNs trained by the different training samples, which consist of the neutrino and three backgrounds at different rates. The term after \pm is the statistical error of the identification efficiencies. The numbers of the train samples are 24000, respectively. The 3000 events each of the uncorrelated background, fast neutron and ${ }^{8} \mathrm{He} /{ }^{9} \mathrm{Li}$ are regarded as the test sample.

\begin{tabular}{|c|c|c|c|c|}
\hline neutrino rate (\%) & 50.0 & 50.0 & 54.5 & 57.1 \\
uncorrelated background rate (\%) & 16.7 & 12.5 & 9.1 & 9.5 \\
fast neutron rate (\%) & 16.7 & 25.0 & 27.3 & 23.8 \\
${ }^{8} \mathrm{He} /{ }^{9} \mathrm{Li}$ rate (\%) & 16.7 & 12.5 & 9.1 & 9.5 \\
\hline neutrino eff.(\%) & $94.1 \pm 0.43$ & $90.5 \pm 0.54$ & $92.6 \pm 0.48$ & $93.7 \pm 0.44$ \\
uncorrelated background eff.(\%) & $98.3 \pm 0.24$ & $98.1 \pm 0.25$ & $96.4 \pm 0.34$ & $96.7 \pm 0.33$ \\
fast neutrons eff.(\%) & $29.3 \pm 0.83$ & $35.8 \pm 0.88$ & $34.6 \pm 0.87$ & $32.8 \pm 0.86$ \\
${ }^{8} \mathrm{He} /{ }^{9} \mathrm{Li}$ eff.(\%) & $91.3 \pm 0.51$ & $90.6 \pm 0.53$ & $87.7 \pm 0.60$ & $87.5 \pm 0.60$ \\
\hline
\end{tabular}




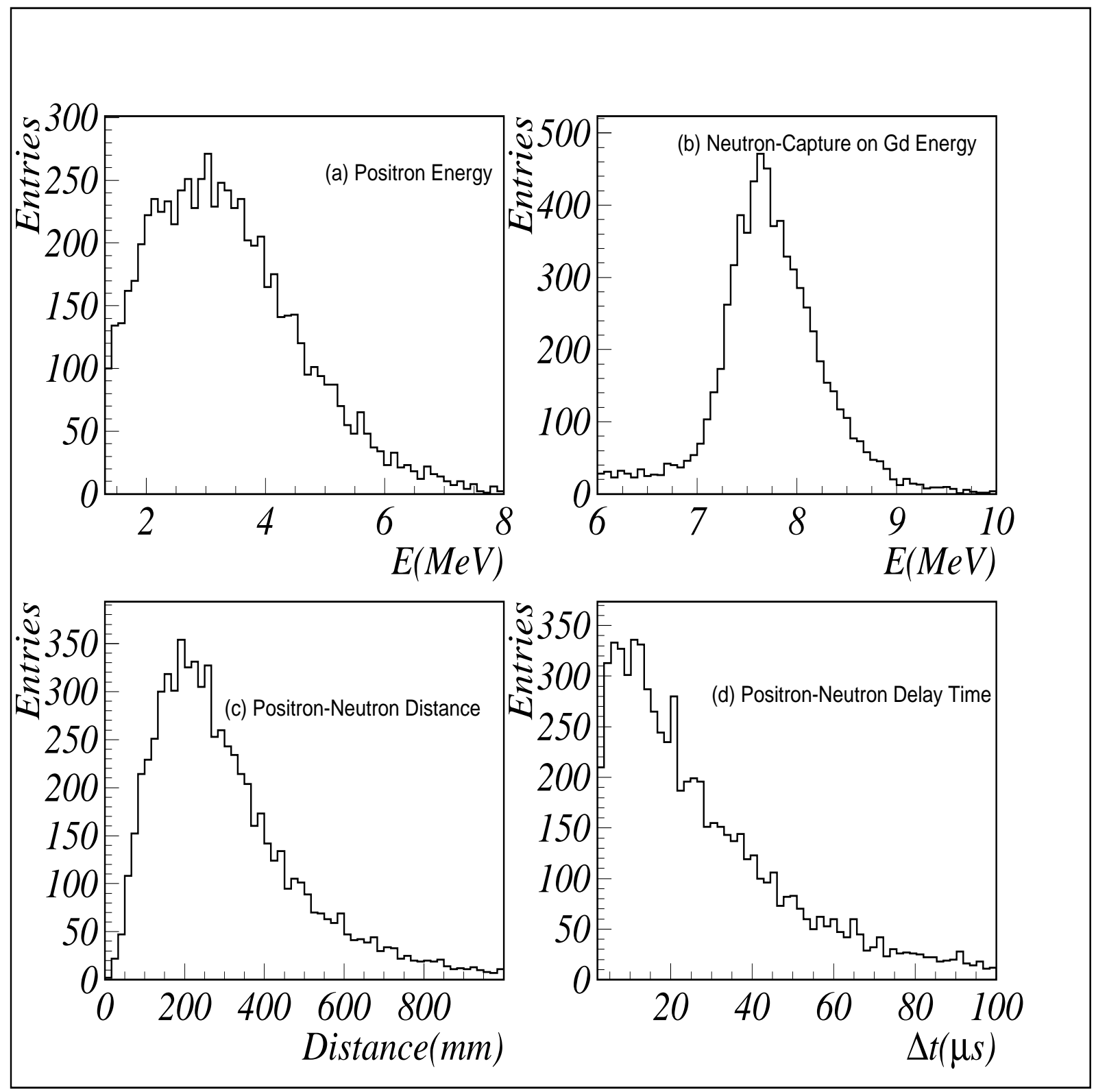

Fig. 1: The neutrino events for the Monte-Carlo simulation of the toy detector are uniformly generated throughout Gd-LS region. (a) is the distribution of the positron energy; (b) is the distribution of the energy of the neutron captured by $\mathrm{Gd}$; (c) is the distribution of the distance between the positron and neutron positions; (d) is the distribution of the delay time of the neutron signal. 